\title{
CANONICAL FORMS AND INTEGRABILITY OF BiHAMILTONIAN SYSTEMS
}

\author{
By \\ Peter J. Olver
}

IMA Preprint Series \# 641

May 1990 


\title{
Canonical Forms and Integrability of BiHamiltonian Systems
}

\author{
Peter J. Olvert \\ School of Mathematics \\ University of Minnesota \\ Minneapolis, MN \\ USA \\ 55455
}

\begin{abstract}
Turiel's complete list of canonical forms for finite-dimensional, nondegenerate, compatible pairs of Hamiltonian structures is used to determine the precise local integrability of biHamiltonian systems of ordinary differential equations. Also, classification of incompatible Hamiltonian pairs in four dimensions and the relationship between compatibility and integrability are discussed.
\end{abstract}

$\dagger$ Research supported in part by NSF Grant DMS 89-01600.

$3 / 22 / 90$ 
A system of differential equations is called biHamiltonian, [8], [11], if it can be written in Hamiltonian form in two distinct ways:

$$
\dot{\mathrm{x}}=\mathrm{J}_{1} \nabla \mathrm{H}_{1}=\mathrm{J}_{2} \nabla \mathrm{H}_{0}, \quad \mathrm{x} \in \mathrm{M} .
$$

Here $M$ is a real or complex $n$-dimensional manifold, $\mathrm{H}_{0}(\mathrm{x}), \mathrm{H}_{1}(\mathrm{x})$ are the two Hamiltonian functions, and $\mathrm{J}_{1}(\mathrm{x}), \mathrm{J}_{2}(\mathrm{x})$ are skew symmetric $\mathrm{n} \times \mathrm{n}$ Hamiltonian matrices, not constant multiples of each other, determining Poisson brackets on M:

$$
\{\mathrm{F}, \mathrm{G}\}_{\mathrm{v}}=\nabla \mathrm{F}^{\mathrm{T}} \mathrm{J}_{\mathrm{v}}(\mathrm{x}) \nabla \mathrm{G}, \quad \mathrm{v}=1,2 .
$$

The Jacobi identity requires that each $\mathrm{J}_{\mathrm{v}}(\mathrm{x})$ satisfy a quadratically non-linear system of partial differential equations, cf. [11; Proposition 6.8]. We call the structure defined by $\mathrm{J}_{1}, \mathrm{~J}_{2}$ a Hamiltonian pair. The Hamiltonian pair is compatible if the sum $\mathrm{J}_{1}(\mathrm{x})+\mathrm{J}_{2}(\mathrm{x})$ also determines a Poisson bracket, which, owing to the quadratic nature of the Jacobi identity, implies that any constant coefficient linear combination of $\mathrm{J}_{1}$ and $\mathrm{J}_{2}$ is also a valid Poisson bracket. In the symplectic case, each $\mathrm{J}_{\mathrm{v}}(\mathrm{x})$ is nonsingular (so $\mathrm{n}$ is necessarily even), and the nonlinear Jacobi conditions can be replaced by the linear condition that the symplectic two forms

$$
\Omega_{v}=\frac{1}{2} \mathrm{dx}^{\mathrm{T}} \wedge \mathrm{K}_{v}(\mathrm{x}) \mathrm{dx}, \quad \mathrm{K}_{v}(\mathrm{x})=\mathrm{J}_{\mathrm{v}}(\mathrm{x})^{-1},
$$

are closed, i.e. $d \Omega_{v}=0$. Compatibility is equivalent to the closure of the two-form

$$
\frac{1}{2} d x^{T} \wedge\left(K_{1}(x)^{-1}+K_{2}(x)^{-1}\right)^{-1} d x .
$$

According to the fundamental theorem of Magri, [8], [11; Theorem 7.24], provided certain technical hypotheses hold, biHamiltonian systems for compatible Hamiltonian pairs are completely integrable.

Theorem 1. Suppose $J_{1}, J_{2}$ form a compatible Hamiltonian pair, with $J_{1}$ symplectic. For each associated biHamiltonian system (1), there exists a hierarchy of Hamiltonian functions $\mathrm{H}_{0}, \mathrm{H}_{1}, \mathrm{H}_{2}, \mathrm{H}_{3}, \ldots$, all in involution with respect to either Poisson bracket, $\left\{\mathrm{H}_{\mathrm{j}}, \mathrm{H}_{\mathrm{k}}\right\}_{v}=0$, generating mutually commuting biHamiltonian flows

$$
\dot{\mathrm{x}}=\mathrm{J}_{1} \nabla \mathrm{H}_{\mathrm{k}}=\mathrm{J}_{2} \nabla \mathrm{H}_{\mathrm{k}-1} \text {. }
$$


Thus such biHamiltonian systems are completely integrable in the classical sense provided enough of the Hamiltonians in the associated hierarchy are functionally independent. The goals of the present paper are two: first to investigate in more detail the integrability of particular biHamiltonian systems; second, to determine the proper role that the compatibility condition plays in the integrability of such systems. Both investigations will rest on the determination of canonical forms for such Hamiltonian pairs, and then explicitly determining all associated biHamiltonian systems. Note that for a given pair, the corresponding biHamiltonian systems are found by solving the linear system of partial differential equations

$$
\nabla \mathrm{H}_{0}=\mathrm{M} \nabla \mathrm{H}_{1}, \quad \mathrm{M}=\mathrm{J}_{2}^{-1} \cdot \mathrm{J}_{1}=\mathrm{K}_{2} \cdot \mathrm{K}_{1}^{-1},
$$

where $\mathrm{M}$ is the transpose of the recursion operator, [11]. We remark here that the simple system of differential equations (5), which arises in a surprising number of different contexts, is not well understood, except in the particular case when the matrix $\mathrm{M}$ is constant, in which case the general solution can be found in [7].

A Hamiltonian pair is called non-degenerate at the point $\mathrm{x}$ if the skew-symmetric matrix pencil

$$
\mathrm{J}_{\lambda}(\mathrm{x}) \equiv \mathrm{J}_{2}(\mathrm{x})-\lambda \mathrm{J}_{1}(\mathrm{x}), \quad \lambda \in \mathbb{C} \cup\{\infty\},
$$

is non-singular for at least one (and hence for all but a finite number of) $\lambda$. (For $\lambda=\infty$, set $J_{\lambda}(x)=J_{1}(x)$.) In this case, the $\lambda^{\prime}$ 's for which $J_{\lambda}(x)$ is singular are called the eigenvalues of the pair. If the pair is compatible, and non-degenerate, we can assume without loss of generality that $\mathrm{J}_{1}$ is symplectic, i.e. $\lambda=\infty$ is not an eigenvalue. The complete algebraic type of a non-degenerate pair of skew-symmetric matrices is given by the elementary divisors and Segre characteristic of the matrix pencil $J_{\lambda}(x)$, cf. [6]. (Degenerate pairs of skew-symmetric matrices are handled by the more detailed Kronecker theory.) A pair is called elementary at $\mathrm{x}$ if it has just one complex eigenvalue. A nondegenerate pair is called irreducible at $\mathrm{x}$ if it has Segre characteristic [(nn)], (analogous to a single Jordan block), so every elementary pair is the direct sum of irreducible pairs all having the same eigenvalue. Every nondegenerate complex skew symmetric matrix pencil is algebraically the direct sum of irreducible skew symmetric matrix pencils.

Theorem 2. The eigenvalues, elementary divisors and Segre characteristic of a Hamiltonian pair are invariant under the flow of any associated biHamiltonian system. 
Definition 3. A (non-degenerate) Hamiltonian pair is generic on a domain $M$ if it has constant Segre characteristic over all of M, i.e. the algebraic type of the pair does not vary from point to point, and also the number of functionally independent eigenvalues does not vary, i.e. the dimension of the subspace of $\left.\mathrm{T}^{*} \mathrm{M}\right|_{\mathrm{x}}$ spanned by their differentials $\left\{\mathrm{d} \lambda_{1}, \ldots, \mathrm{d} \lambda_{l}\right\}$ is independent of $\mathrm{x}$. (In particular, each eigenvalue $\lambda_{\mathrm{m}}$ is either constant or has no critical points on $\mathrm{M}$.)

Non-generic points are singularities of the pair, and must be handled by more sophisticated techniques; see [2] for the case of a single Poisson structure in the plane. According to Theorem 2, as far as the flow of any biHamiltonian system is concerned, we can safely restrict our attention to a domain where the pair is generic.

According to the results of Turiel, [14], [15], any complex-analytic generic nondegenerate compatible Hamiltonian pair can be locally expressed as the Cartesian product of elementary pairs, each having just one eigenvalue. (Turiel's classification results extend to real Hamiltonian pairs, as do our classifications of biHamiltonian systems, but, for simplicity, we will restrict our attention here to complex analytic systems.) In the case of constant eigenvalue, any elementary pair is in turn the Cartesian product of irreducible pairs; however, this does not hold in the case of non-constant eigenvalue. We will present the details of the Turiel classification and the structure of associated biHamiltonian systems in five stages, corresponding to

I) irreducible, constant eigenvalue pairs,

II) elementary, constant eigenvalue pairs,

III) irreducible, non-constant eigenvalue pairs,

IV) elementary, non-constant eigenvalue pairs,

V) general generic, compatible, non-degenerate Hamiltonian pairs.

We assume that neither 0 nor $\infty$ is an eigenvalue, so the Hamiltonian pair is determined by two compatible symplectic forms. Darboux' theorem, [11; Theorem 6.22], implies that we can write the first symplectic form in canonical form

$$
\Omega_{1}=\mathrm{dp}_{0} \wedge \mathrm{dq}_{0}+\mathrm{dp}_{1} \wedge \mathrm{dq}_{1}+\ldots+\mathrm{dp}_{\mathrm{n}} \wedge \mathrm{dq}_{\mathrm{n}} .
$$

Therefore, the problem reduces to how to place the second form $\Omega_{2}$ into a canonical form using canonical transformations of the phase space. 


\section{I) Irreducible, Constant Eigenvalue Pairs,}

Theorem 4. Any irreducible compatible Hamiltonian pair with constant nonzero eigenvalue $\lambda$ has the canonical form

$$
\Omega_{2}=\mu \Omega_{1}+d p_{0} \wedge d q_{1}+d p_{1} \wedge d q_{2}+\ldots+d p_{n-1} \wedge d q_{n}
$$

where $\Omega_{1}$ is given by (7), and $\mu=\lambda^{-1}$.

The associated symplectic matrices for the pair (7), (8) are

$$
\mathrm{K}_{1}=\left(\begin{array}{cc}
0 & \mathrm{I} \\
-\mathrm{I} & 0
\end{array}\right), \quad \mathrm{K}_{2}=\left(\begin{array}{cc}
0 & \mu \mathrm{I}+\mathrm{U} \\
-\mu \mathrm{I}-\mathrm{U}^{\mathrm{T}} & 0
\end{array}\right) \text {. }
$$

which is the canonical algebraic form of Weierstass for a nondegenerate pair of skewsymmetric complex matrices, [6]. Here $I$ is the $(n+1) \times(n+1)$ identity matrix, and $U$ is the upper triangular matrix of the same size with 1 's on the super-diagonal and 0 's elsewhere. Inverting the matrices (9) will give a canonical form for the Hamiltonian matrices $\mathrm{J}_{\mathrm{v}}$, although these are somewhat complicated to work with directly. However, this pair assumes the simpler canonical form

$$
\mathrm{J}_{1}=\left(\begin{array}{cc}
0 & \mathrm{I} \\
-\mathrm{I} & 0
\end{array}\right), \quad \mathrm{J}_{2}=\left(\begin{array}{cc}
0 & \lambda \mathrm{I}+\mathrm{U} \\
-\lambda \mathrm{I}-\mathrm{U}^{\mathrm{T}} & 0
\end{array}\right)
$$

under the canonical transformation $(p, q) \rightarrow\left(A^{T} p, A^{-1} q\right)$, where the matrix $A$ is such that $A(\mu I+U)^{-1} A^{-1}=\lambda I+U$ is in Jordan canonical form. The general form for any associated biHamiltonian system follows from the solution to (5) using the methods of [7].

Theorem 5. Suppose $\mathrm{H}_{1}(\mathbf{x}), \mathrm{H}_{0}(\mathbf{x}), \mathbf{x}=(\mathbf{p}, \mathbf{q})$, are analytic functions which define a biHamiltonian system (1) relative to the canonical irreducible constant eigenvalue Hamiltonian pair (7), (8) on a convex open subset. Then there exist scalar-valued functions $\mathrm{F}_{\mathrm{k}}(\xi, \eta), \mathrm{k}=0, \ldots, \mathrm{n}$, such that

$\mathrm{H}_{0}(\mathbf{x})=\mathrm{H}_{0}^{(0)}(\mathbf{x})+\mathrm{H}_{0}^{(1)}(\mathbf{x})+\ldots+\mathrm{H}_{0}^{(\mathrm{n})}(\mathbf{x}), \quad \mathrm{H}_{1}(\mathbf{x})=\mathrm{H}_{1}^{(0)}(\mathbf{x})+\mathrm{H}_{1}^{(1)}(\mathbf{x})+\ldots+\mathrm{H}_{1}^{(\mathrm{n})}(\mathbf{x})$,

with 


$$
\begin{aligned}
& H_{\delta}^{(k)}(x)=\left.\mu \frac{\partial^{k}}{\partial s^{k}} F_{k}(\pi(s), \varpi(s))\right|_{s=0}+\left.k \frac{\partial^{k-1}}{\partial s^{k-1}} F_{k}(\pi(s), \varpi(s))\right|_{s=0}, \\
& H_{1}^{(k)}(x)=\left.\frac{\partial^{k}}{\partial s^{k}} F_{k}(\pi(s), \varpi(s))\right|_{s=0},
\end{aligned}
$$

where

$$
\begin{aligned}
& \pi(s)=p_{0}+s p_{1}+s^{2} p_{2}+\ldots+s^{n} p_{n}, \\
& \varpi(s)=q_{n}+s q_{n-1}+s^{2} q_{n-2}+\ldots+s^{n} q_{0} .
\end{aligned}
$$

Example 6. Consider the case $n=2$. Then

$$
\pi(\mathrm{s})=\mathrm{p}_{0}+\mathrm{s} \mathrm{p}_{1}+\mathrm{s}^{2} \mathrm{p}_{2}, \quad \sigma(\mathrm{s})=\mathrm{q}_{2}+\mathrm{s} \mathrm{q}_{1}+\mathrm{s}^{2} \mathrm{q}_{0} .
$$

Substituting into (11), we find that the general solution to (5) in this case is a sum of three particular types of solution:

$$
\begin{array}{ll}
\mathrm{H}_{0}^{(0)}=\mu \mathrm{F}_{0}\left(\mathrm{p}_{0}, \mathrm{q}_{2}\right), & \mathrm{H}_{1}^{(0)}=\mathrm{F}_{0}\left(\mathrm{p}_{0}, \mathrm{q}_{2}\right) \\
\mathrm{H}_{0}^{(1)}=\mu \mathrm{H}_{1}^{(1)}+\mathrm{F}_{1}\left(\mathrm{p}_{0}, \mathrm{q}_{2}\right), & \mathrm{H}_{1}^{(1)}=\frac{\partial \mathrm{F}_{1}}{\partial \mathrm{p}_{0}} \mathrm{p}_{1}+\frac{\partial \mathrm{F}_{1}}{\partial \mathrm{q}_{2}} \mathrm{q}_{1}, \\
\mathrm{H}_{0}^{(2)}=\mu \mathrm{H}_{1}^{(2)}+2 \frac{\partial \mathrm{F}_{2}}{\partial \mathrm{p}_{0}} \mathrm{p}_{2}+2 \frac{\partial \mathrm{F}_{2}}{\partial \mathrm{q}_{2}} \mathrm{q}_{0}, \\
\mathrm{H}_{1}^{(2)}=\frac{\partial^{2} \mathrm{~F}_{2}}{\partial \mathrm{p}_{0}^{2}} \mathrm{p}_{1}^{2}+2 \frac{\partial^{2} \mathrm{~F}_{2}}{\partial \mathrm{p}_{0} \partial \mathrm{q}_{2}} \mathrm{p}_{1} \mathrm{q}_{1}+\frac{\partial^{2} \mathrm{~F}_{2}}{\partial \mathrm{q}_{2}^{2}} \mathrm{q}_{1}^{2}+2 \frac{\partial \mathrm{F}_{2}}{\partial \mathrm{p}_{0}} \mathrm{p}_{2}+2 \frac{\partial \mathrm{F}_{2}}{\partial \mathrm{q}_{2}} \mathrm{q}_{0},
\end{array}
$$

where $\mathrm{F}_{0}, \mathrm{~F}_{1}, \mathrm{~F}_{2}$ are arbitrary functions of the variables $\mathrm{p}_{0}, \mathrm{q}_{2}$.

Similarly, it can be shown that the general Hamiltonians determined by (11) are polynomials in the minor variables $\mathrm{p}_{1}, \ldots, \mathrm{p}_{\mathrm{n}}, \mathrm{q}_{0}, \ldots, \mathrm{q}_{\mathrm{n}-1}$, whose coefficients are certain derivatives of the arbitrary smooth functions $\mathrm{F}_{\mathrm{k}}\left(\mathrm{p}_{0}, \mathrm{q}_{\mathrm{n}}\right)$ of the remaining two major variables, $\mathrm{p}_{0}, \mathrm{q}_{\mathrm{n}}$. In outline, the proof of this and similar subsequent results proceeds in two steps. First one verifies by direct, elementary computation that (11) really do define solutions to the system (5). Then, to show that these are the only solutions, we cross differentiate to deduce that the general solution must be an affine function of the top order 
minor variables $p_{n}, q_{0}$, and, moreover, the coefficients of these variables can be matched by a suitable choice of the solution (11) for $k=n$. The linearity of (5) and an easy induction will complete the argument.

Theorem 5 demonstrates the complete integrability of any biHamiltonian system corresponding to an irreducible, constant eigenvalue Hamiltonian pair. Indeed, the subsystem governing the time evolution of the major variables is the autonomous twodimensional (one degree of freedom) Hamiltonian system with Hamiltonian $n ! F_{n}\left(p_{0}, q_{n}\right)$,

$$
\frac{\mathrm{dp}_{0}}{\mathrm{dt}}=-\frac{\partial \mathrm{H}_{1}}{\partial \mathrm{q}_{0}}=-\mathrm{n} ! \frac{\partial \mathrm{F}_{\mathrm{n}}}{\partial \mathrm{q}_{\mathrm{n}}}, \quad \frac{\mathrm{dq}_{\mathrm{n}}}{\mathrm{dt}}=\frac{\partial \mathrm{H}_{1}}{\partial \mathrm{p}_{\mathrm{n}}}=\mathrm{n} ! \frac{\partial \mathrm{F}_{\mathrm{n}}}{\partial \mathrm{p}_{0}},
$$

and is easily integrated by quadrature. (Curiously, the canonically conjugate variables $\mathrm{p}_{0}, \mathrm{q}_{\mathrm{n}}$ for the reduced system (13) are not canonically conjugate for the standard symplectic structure given by $\Omega_{1}$, nor are they conjugate for $\Omega_{2}$.) The time evolution of the minor variables is then determined by successively solving a hierarchy of twodimensional forced linear Hamiltonian systems in the variables $p_{k}, q_{n-k}$, each of the form

$$
\begin{aligned}
\frac{d p_{k}}{d t} & =-n !\left(\frac{\partial^{2} F_{n}}{\partial p_{0} \partial q_{n}} p_{k}+\frac{\partial^{2} F_{n}}{\partial q_{n}^{2}} q_{n-k}\right)-G_{k}(t), \\
\frac{d q_{n-k}}{d t} & =n !\left(\frac{\partial^{2} F_{n}}{\partial p_{0}^{2}} p_{k}+\frac{\partial^{2} F_{n}}{\partial p_{0} \partial q_{n}} q_{n-k}\right)+\widetilde{G}_{k}(t),
\end{aligned}
$$

where $G_{k}, \widetilde{G}_{k}$ are certain explicit functions of $\left(p_{0}, \ldots, p_{k-1}, q_{n}, \ldots, q_{n-k+1}\right)$, whose time evolution has thus already been determined. We conclude that any biHamiltonian system for an irreducible, constant eigenvalue Hamiltonian pair can be integrated by solving a single two-dimensional autonomous Hamiltonian system, followed by a sequence of forced linear, nonautonomous two-dimensional Hamiltonian systems.

\section{Elementary, Constant Eigenvalue Pairs.}

Theorem 7. Every compatible Hamiltonian pair with constant eigenvalue(s) is (locally) the Cartesian product of irreducible pairs.

We can therefore introduce local coordinates 


$$
\mathbf{x}=(\mathbf{p}, \mathbf{q})=\left(\mathbf{p}^{1}, \ldots, \mathbf{p}^{\mathrm{m}}, \mathbf{q}^{1}, \ldots, \mathbf{q}^{\mathrm{m}}\right), \quad \mathbf{p}^{\mathrm{i}}=\left(\mathrm{p}_{0}^{\mathrm{i}}, \ldots, \mathrm{p}_{\mathrm{n}_{\mathrm{i}}}^{\mathrm{i}}\right), \quad \mathbf{q}^{\mathrm{i}}=\left(\mathrm{q}_{0}^{\mathrm{i}}, \ldots, \mathrm{q}_{n_{\mathrm{i}}}^{\mathrm{i}}\right)
$$

such that $\Omega_{v}=\Omega_{v}^{1}+\ldots+\Omega_{v}^{m}$, where each $\left(2 n_{i}+2\right)$-dimensional subpair $\Omega_{1}^{\mathrm{i}}$, $\Omega_{2}^{\mathrm{i}}$ is irreducible, compatible, taking the canonical form (7), (8) in its coordinates $\left(\mathbf{p}^{\mathrm{i}}, \mathbf{q}^{\mathrm{i}}\right)$. Restricting attention to the case of just one eigenvalue, assume that the subpairs are arranged in order of size: $n_{1} \geq n_{2} \geq \ldots \geq n_{m}$. For $k \geq 0$, let $m_{k}$ denote the number of irreducible sub-pairs of dimension greater than $2 k+1$, which, according to our ordering, will be the first $\mathrm{m}_{\mathrm{k}}$ subpairs. In particular $\mathrm{m}_{0}=\mathrm{m}$.

Theorem 8. The most general biHamiltonian system for a canonical elementary Hamiltonian pair with constant eigenvalue $\lambda=\mu^{-1}$ has the form (1) with

$$
\mathrm{H}_{0}(\mathbf{x})=\mathrm{H}_{0}^{(0)}(\mathbf{x})+\mathrm{H}_{0}^{(1)}(\mathbf{x})+\ldots \mathrm{H}_{\delta}^{\left(\mathrm{n}_{1}\right)}, \quad \mathrm{H}_{1}(\mathbf{x})=\mathrm{H}_{1}^{(0)}(\mathbf{x})+\mathrm{H}_{1}^{(1)}(\mathbf{x})+\ldots \mathrm{H}_{1}^{\left(\mathrm{n}_{1}\right)},
$$

where

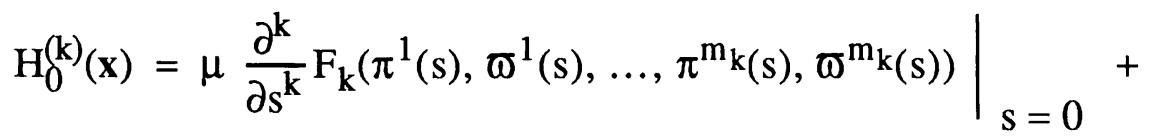

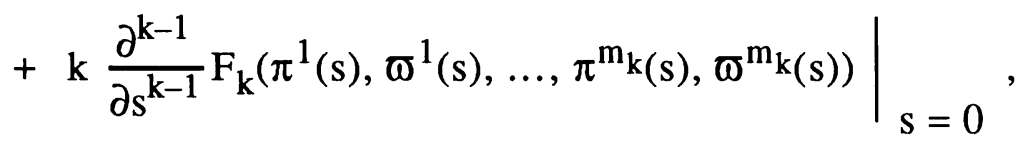

$$
\begin{aligned}
& \mathrm{H}_{1}^{(\mathrm{k})}(\mathbf{x})=\left.\frac{\partial^{\mathrm{k}}}{\partial \mathrm{s}^{\mathrm{k}}} \mathrm{F}_{\mathrm{k}}\left(\pi_{1}(\mathrm{~s}), \varpi_{1}(\mathrm{~s}), \ldots, \pi_{\mathrm{m}_{\mathrm{k}}}(\mathrm{s}), \varpi_{\mathrm{m}_{\mathrm{k}}}(\mathrm{s})\right)\right|_{\mathrm{s}=0} .
\end{aligned}
$$

Here $\pi^{\mathrm{i}}(\mathrm{s}), \varpi^{\mathrm{i}}(\mathrm{s})$ are the parametrized variables for the $\mathrm{i}^{\text {th }}$ sub-pair, given by the expressions (12), with $n=n_{i}$, and the additional index $i$ on all the variables $p_{j}^{i}, q_{j}^{i}$.

As in the irreducible case, we find that these Hamiltonians are polynomials in the minor variables $p_{j}^{i}, q_{n_{i}-j}^{i}, j \geq 1$, wose coefficients are certain derivatives of arbitrary functions of the major variables $\mathrm{p}_{0}^{\mathrm{i}}, \mathrm{q}_{\mathrm{n}_{\mathrm{i}}}^{\mathrm{i}}$. Therefore, to solve such a biHamiltonian system, we must integrate an autonomous m-dimensional Hamiltonian system in the major variables, followed by a sequence of linear non-autonomous Hamiltonian systems in the appropriate minor variables $p_{k}^{i}, q_{n_{i}-k}^{i}$, for all $i$ with $n_{i} \geq k \geq 1$. 


\section{Irreducible, Non-constant Eigenvalue Pairs.}

Theorem 9. A generic irreducible compatible Hamiltonian pair with nonconstant eigenvalue $\lambda=1 / \mathrm{p}_{0}$ can be put into the canonical form

$$
\Omega_{1}=\sum_{i=0}^{n}{d p_{i}} \wedge d q_{i}, \quad \Omega_{2}=\sum_{j+k=l} p_{j}{d p_{k}}_{k} \wedge d q_{l} .
$$

The sum in $\Omega_{2}$ is over all $\mathrm{j}, \mathrm{k}, \mathcal{L}$ from 0 to $\mathrm{n}$ satisfying $\mathrm{j}+\mathrm{k}=\mathcal{l}$.

The coordinates employed here are different from those in Turiel, [14], although the transformation between the two is not hard. This particular bi-Hamiltonian structure has several remarkable properties. Define the $(n+1) \times(n+1)$ banded upper triangular matrix

$$
P_{n}(\mathbf{p})=P(\mathbf{p})=\left(\begin{array}{cccccc}
\mathrm{p}_{0} & \mathrm{p}_{1} & \mathrm{p}_{2} & \mathrm{p}_{3} & \cdots & \mathrm{p}_{\mathrm{n}} \\
& \mathrm{p}_{0} & \mathrm{p}_{1} & \mathrm{p}_{2} & \cdots & \mathrm{p}_{\mathrm{n}-1} \\
& & \mathrm{p}_{0} & \mathrm{p}_{1} & \cdots & \cdots \\
& & & \mathrm{p}_{0} & \cdots & \cdots \\
& & & & \cdots & \cdots \\
& & & & & \mathrm{p}_{0}
\end{array}\right) \text {. }
$$

Then the skew-symmetric matrix giving the symplectic two-form $\Omega_{2}$ is

$$
K_{2}(p)=\left(\begin{array}{cc}
0 & P(p) \\
-P(p)^{T} & 0
\end{array}\right) .
$$

The Hamiltonian matrix $\mathrm{J}_{2}(\mathrm{p})=\mathrm{K}_{2}(\mathrm{p})^{-1}$ is much more complicated, involving the inverse $\mathrm{R}(\mathbf{p})=\mathrm{P}(\mathbf{p})^{-1}$, which has the same banded upper triangular form as $\mathrm{P}$ with entries

$$
\mathrm{r}_{0}(\mathrm{p})=\frac{1}{\mathrm{p}_{0}}, \quad \mathrm{r}_{\mathrm{k}}(\mathrm{p})=\sum_{\substack{\mathrm{i}_{1}+\mathrm{i}_{2}+\ldots+\mathrm{i}_{\tau}=k \\ \mathrm{i}_{v} \geq 1}}(-1)^{\tau+1} \frac{\mathrm{p}_{\mathrm{i}_{1}} \mathrm{p}_{\mathrm{i}_{2}} \cdots \mathrm{p}_{\mathrm{i}_{\tau}}}{\mathrm{p}_{0}^{\tau+1}}, \quad \mathrm{k} \geq 1
$$

However, the explicit change of coordinates

$$
\tilde{\mathbf{p}}=\mathbf{r}(\mathbf{p}), \quad \tilde{\mathbf{q}}=\mathrm{P}(\mathbf{p})^{2} \mathbf{q},
$$


is a canonical involution, which maps $P$ to $P(\tilde{\mathbf{p}})=P(\mathbf{p})^{-1}$, and so changes the second Hamiltonian matrix into its inverse. Thus the pair has the alternative canonical form

$$
\mathrm{J}_{1}=\left(\begin{array}{cc}
0 & \mathrm{I} \\
-\mathrm{I} & 0
\end{array}\right), \quad \mathrm{J}_{2}=\left(\begin{array}{cc}
0 & \mathrm{P}(\tilde{\mathbf{p}}) \\
-\mathrm{P}(\tilde{\mathbf{p}})^{\mathrm{T}} & 0
\end{array}\right) \text {, }
$$

with eigenvalue $\lambda=\widetilde{p}_{0}$. Therefore, as in the constant eigenvalue case, we conclude that both $\mathrm{P}(\mathbf{p})$ and its inverse determine isomorphic Hamiltonian matrices!

Theorem 10. Suppose $\mathrm{H}_{1}(\mathbf{x}), \mathrm{H}_{0}(\mathbf{x}), \mathbf{x}=(\mathbf{p}, \mathbf{q})$, are analytic functions which define a biHamiltonian system relative to the canonical irreducible non-constant eigenvalue Hamiltonian pair (17) on a convex open subset. Then there exist scalar-valued functions $\mathrm{h}(\xi), \tilde{\mathrm{h}}(\xi), \mathrm{F}_{\mathrm{k}}(\xi, \eta), \mathrm{k}=0, \ldots, \mathrm{n}-1$, such that

$\mathrm{H}_{0}(\mathbf{x})=\tilde{\mathrm{h}}\left(\mathrm{p}_{1}\right)+\mathrm{H}_{0}^{(0)}(\mathbf{x})+\ldots+\mathrm{H}_{\delta}^{(\mathrm{n}-1)}(\mathbf{x}), \quad \mathrm{H}_{1}(\mathbf{x})=\mathrm{h}\left(\mathrm{p}_{1}\right)+\mathrm{H}_{1}^{(0)}(\mathbf{x})+\ldots+\mathrm{H}_{1}^{(\mathrm{n}-1)}(\mathbf{x})$,

where $\tilde{h}^{\prime}(\xi)=\xi h^{\prime}(\xi)$, and

$$
\begin{aligned}
& H_{\delta}^{(k)}(x)=\left.\frac{\partial^{k}}{\partial s^{k}}\left\{\pi(s) \pi^{\prime}(s) F_{k}(\pi(s), \varpi(s))\right\}\right|_{s=0}, \\
& H_{1}^{(k)}(x)=\left.\frac{\partial^{k}}{\partial s^{k}}\left\{\pi^{\prime}(s) F_{k}(\pi(s), \varpi(s))\right\}\right|_{s=0} .
\end{aligned}
$$

Here $\pi(s), \sigma(s)$ are given by (12), and $\pi^{\prime}(s)$ is the derivative of $\pi$ with respect to $s$.

Example 11. If $\mathrm{n}=2$, the general biHamiltonian system is given by a sum of the following three particular types of Hamiltonians:

$$
\begin{aligned}
& \mathrm{H}_{0}^{(0)}=\tilde{\mathrm{h}}\left(\mathrm{p}_{0}\right), \quad \mathrm{H}_{1}^{(0)}=\mathrm{h}\left(\mathrm{p}_{0}\right), \quad \tilde{\mathrm{h}}^{\prime}\left(\mathrm{p}_{0}\right)=\mathrm{p}_{0} \mathrm{~h}^{\prime}\left(\mathrm{p}_{0}\right), \\
& \mathrm{H}_{0}^{(1)}=\mathrm{p}_{0} \mathrm{p}_{1} \mathrm{~F}_{0}\left(\mathrm{p}_{0}, \mathrm{q}_{2}\right), \quad \mathrm{H}_{1}^{(1)}=\mathrm{p}_{1} \mathrm{~F}_{0}\left(\mathrm{p}_{0}, \mathrm{q}_{2}\right), \\
& \mathrm{H}_{0}^{(2)}=\left(2 \mathrm{p}_{0} \mathrm{p}_{2}+\mathrm{p}_{1}^{2}\right) \mathrm{F}_{1}\left(\mathrm{p}_{0}, \mathrm{q}_{2}\right)+\mathrm{p}_{0} \mathrm{p}_{1}^{2} \frac{\partial \mathrm{F}_{1}}{\partial \mathrm{p}_{0}}+\mathrm{p}_{0} \mathrm{p}_{1} \mathrm{q}_{1} \frac{\partial \mathrm{F}_{1}}{\partial \mathrm{q}_{2}} \\
& \mathrm{H}_{1}^{(2)}=2 \mathrm{p}_{2} \mathrm{~F}_{1}\left(\mathrm{p}_{0}, \mathrm{q}_{2}\right)+\mathrm{p}_{1}^{2} \frac{\partial \mathrm{F}_{1}}{\partial \mathrm{p}_{0}}+\mathrm{p}_{1} \mathrm{q}_{1} \frac{\partial \mathrm{F}_{1}}{\partial \mathrm{q}_{2}}
\end{aligned}
$$


In general, note that by Theorem 2 , the eigenvalue $\lambda$ is a constant, hence $\mathrm{p}_{0}$ is a first integral for such Hamiltonian systems. Once its value is fixed, the other minor variable, $\mathrm{q}_{2}$, is found by solving a single autonomous ordinary differential equation. The minor variables satisfy a sequence of forced, linear planar Hamiltonian systems.

\section{Elementary, Non-constant Eigenvalue Pairs.}

Theorem 12. Any generic elementary compatible Hamiltonian pair with nonconstant eigenvalue $\lambda=1 / \mathrm{p}_{0}$ can be written in the canonical form

$$
\begin{aligned}
& \Omega_{1}=\mathrm{dp}_{0} \wedge \mathrm{dq}_{0}+\sum_{\mathrm{i}=1}^{\mathrm{m}} \sum_{\mathrm{j}=1}^{\mathrm{n}_{\mathrm{i}}} \mathrm{dp}_{\mathrm{j}}^{\mathrm{i}} \wedge d \mathrm{dq}_{\mathrm{j}}^{\mathrm{i}}, \\
& \Omega_{2}=\mathrm{p}_{0} \mathrm{dp}_{0} \wedge d \mathrm{dq}_{0}+\sum_{\mathrm{i}=1}^{\mathrm{m}} \sum_{\substack{j+\mathrm{k}=l \\
j+\mathrm{k}+l \neq 0}} \mathrm{p}_{\mathrm{j}}^{\mathrm{i}} \mathrm{dp}_{\mathrm{k}}^{\mathrm{i}} \wedge d \mathrm{dq}_{\mathcal{L}}^{\mathrm{i}} .
\end{aligned}
$$

Beyond the eigenvalue coorindate $p_{0}$ and its canonical conjugate $q_{0}$, the coordinates come in conjugate sets $\mathbf{p}^{\mathrm{i}}=\left(\mathrm{p}_{1}^{\mathrm{i}}, \ldots, \mathrm{p}_{\mathrm{n}_{\mathrm{i}}}^{\mathrm{i}}\right), \mathbf{q}^{\mathrm{i}}=\left(\mathrm{q}_{1}^{\mathrm{i}}, \ldots, \mathrm{q}_{\mathrm{n}_{\mathrm{i}}}^{\mathrm{i}}\right), \mathrm{i}=1, \ldots, \mathrm{m}$. We also set $\mathrm{p}_{0}^{\mathrm{i}}$ $=p_{0}$ for all $\mathrm{i}$ by convention. The interior sum in $\Omega_{2}$ is over all $\mathrm{j}, \mathrm{k}, \mathcal{l}=0, \ldots, \mathrm{n}_{\mathrm{i}}$ except the case $\mathrm{j}=\mathrm{k}=\ell=0$, which already appears outside the double summation.

Note that this particular pair is algebraically reducible, but not reducible by canonical transformations. The corresponding symplectic matrix for $\Omega_{2}$ is

$$
\mathrm{K}_{2}=\left(\begin{array}{cc}
0 & \mathbf{P}^{*}(\mathbf{p}) \\
-\mathbf{P}^{*}(\mathbf{p})^{\mathrm{T}} & 0
\end{array}\right),
$$

where

$$
\mathbf{P}^{*}(\mathbf{p})=\left(\begin{array}{ccccc}
\mathrm{p}_{0} & \mathbf{p}^{1} & \mathbf{p}^{2} & \ldots & \mathbf{p}^{\mathrm{m}} \\
& \mathrm{P}_{\mathrm{n}_{1}}\left(\hat{\mathbf{p}}^{1}\right) & 0 & \ldots & 0 \\
& & \mathrm{P}_{\mathrm{n}_{2}}\left(\hat{\mathbf{p}}^{2}\right) & & \\
& & & \cdots & \\
& & & & \mathrm{P}_{\mathrm{n}_{\mathrm{m}}}\left(\hat{\mathbf{p}}^{\mathrm{m}}\right)
\end{array}\right)
$$


Here $\hat{\mathbf{p}}^{\mathrm{m}}=\left(\mathrm{p}_{0}, \mathrm{p}_{1}^{\mathrm{m}}, \ldots, \mathrm{p}_{\mathrm{n}_{\mathrm{m}}}^{\mathrm{m}}\right)$, (recall $\left.\mathrm{p}_{0}^{\mathrm{m}}=\mathrm{p}_{0}\right)$ and the $\mathrm{P}_{\mathrm{n}_{\mathrm{m}}}$ 's are the corresponding upper triangular matrices of size $n_{m} \times n_{m}$ as given in (18). Note that the algebraically irreducible sub-blocks corresponding to just the variables $\hat{\mathbf{p}}^{\mathrm{m}}, \hat{\mathbf{q}}^{\mathrm{m}}$ are isomorphic to the irreducible pair (17), but these subblocks are all entangled since the eigenvalue $\lambda=1 / \mathrm{p}_{0}$ is the same in all cases.

To describe the general form for any associated biHamiltonian system, we let

$$
\begin{aligned}
& \pi^{i}(s)=p_{0}+s p_{1}^{i}+s^{2} p_{2}^{i}+\ldots+s^{n_{i}} p_{n_{i}}^{i}, \\
& \zeta^{i}(s)=p_{1}^{i}+s p_{2}^{i}+\ldots+s^{n_{i}-1} p_{n_{i}}^{i}=s^{-1}\left(\pi^{i}(s)-p_{0}\right), \\
& \omega^{i}(s)=q_{n_{i}}^{i}+s q_{n_{i}-1}^{i}+\ldots+s^{n_{i}-1} q_{1}^{i}, \\
& \mu^{i}(s)=\left.\sum_{n=0}^{n_{i}-1} \frac{s^{n}\left(\zeta^{1}(s)\right)^{n+1}}{(n+1) !} \frac{d^{n}}{d t^{n}} \frac{1}{\left(\zeta^{i}(t)\right)^{n+1}}\right|_{t=0}, \quad i=2, \ldots, m, \\
& \sigma^{i}(s)=q_{n_{i}}^{i}+\left.\sum_{n=0}^{n_{i}-1} \frac{s^{n}\left(\zeta^{1}(s)\right)^{n}}{n !} \frac{d^{n}}{d t^{n}} \frac{1}{\left(\zeta^{i}(t)\right)^{n}} \frac{d \omega^{i}(t)}{d t}\right|_{t=0}, \quad i=2, \ldots, m .
\end{aligned}
$$

Remark. The expansions of $\mu^{\mathrm{i}}$ and $\sigma^{\mathrm{i}}$ involve truncations of the remarkable nonlinear series differential operator

$$
\mathcal{D}=D^{-1}: e^{s \mathrm{D} u}: D=1+\sum_{n=1}^{\infty} \frac{\mathrm{s}^{\mathrm{n}}}{\mathrm{n} !} \mathrm{D}^{\mathrm{n}-1} \mathrm{u}^{\mathrm{n}} \mathrm{D}, \quad \mathrm{D}=\frac{\mathrm{d}}{\mathrm{dt}}
$$

where $\mathrm{u}=\mathrm{u}(\mathrm{t})$. The colons indicate a normal ordering of the non-commuting operators $\mathrm{D}$ and $\mathrm{u}$ analogous to the Wick ordering in quantum mechanics. This operator has the surprising property that it commutes with any analytic function $\Phi(\mathrm{u})$ :

$$
\mathcal{D} \Phi(\mathrm{u})=\Phi(\mathcal{D} \mathrm{u})
$$

See [13] for details and applications of this operator to deriving new derivative identities.

Theorem 13. The most general bi-Hamiltonian system for an elementary, nonconstant eigenvalue generic Hamiltonian pair (24) is given by 
$\mathrm{H}_{0}(\mathbf{x})=\tilde{\mathrm{h}}\left(\mathrm{p}_{0}\right)+\mathrm{H}_{0}^{(0)}(\mathbf{x})+\ldots+\mathrm{H}_{0}^{\left(\mathrm{n}_{1}-1\right)}(\mathbf{x}), \quad \mathrm{H}_{1}(\mathbf{x})=\mathrm{h}\left(\mathrm{p}_{0}\right)+\mathrm{H}_{1}^{(0)}(\mathbf{x})+\ldots+\mathrm{H}_{1}^{\left(\mathrm{n}_{1}-1\right)}(\mathbf{x})$,

where

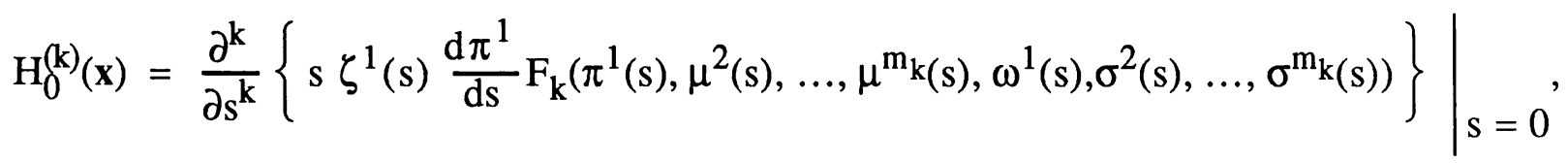

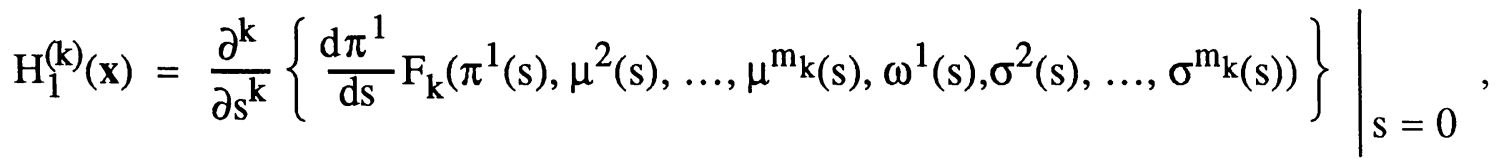

where $h(\xi), \widetilde{h}(\xi), F_{k}\left(\xi_{1}, \ldots, \xi_{m_{k}}, \eta_{1}, \ldots, \eta_{m_{k}}\right), k=0, \ldots, n-2$, are scalar-valued functions, $\tilde{h}^{\prime}(\xi)=\xi h^{\prime}(\xi)$. We assume as before that the blocks are arranged in decreasing order $n_{1} \geq n_{2} \geq \ldots \geq n_{m}$, and $m_{k}$ denotes the number of blocks of size $n_{i} \geq k$.

Example 14. Consider the case of two sub-blocks of size $n_{1}=n_{2}=2$, with coordinates $\left(\mathrm{p}_{0}, \mathrm{p}_{1}, \mathrm{p}_{2}, \mathrm{p}_{1}^{\prime}, \mathrm{p}_{2}^{\prime}, \mathrm{q}_{0}, \mathrm{q}_{1}, \mathrm{q}_{2}, \mathrm{q}_{1}^{\prime}, \mathrm{q}_{2}^{\prime}\right)$. The important variables for (27) are

$$
\begin{array}{ll}
\pi^{1}(\mathrm{~s})=\mathrm{p}_{0}+\mathrm{s}_{1}+\mathrm{s}^{2} \mathrm{p}_{2}, & \omega^{1}(\mathrm{~s})=\mathrm{q}_{2}+\mathrm{sq}_{1}+\mathrm{s}^{2} \mathrm{q}_{0}, \\
\mu(\mathrm{s})=\frac{\mathrm{p}_{1}}{\mathrm{p}_{1}^{\prime}}+\mathrm{s}\left(\frac{\mathrm{p}_{2}}{\mathrm{p}_{1}^{\prime}}-\frac{\mathrm{p}_{1}^{2} \mathrm{p}_{2}^{\prime}}{\mathrm{p}_{1}^{\prime 3}}\right), \quad \sigma(\mathrm{s})=\mathrm{q}_{2}^{\prime}+\mathrm{s} \frac{\mathrm{p}_{1} \mathrm{q}_{1}^{\prime}}{\mathrm{p}_{1}^{\prime}} .
\end{array}
$$

The general pair of Hamiltonians is a sum of the following two:

$$
\begin{aligned}
& \mathrm{H}_{0}^{(0)}=\mathrm{p}_{0} \mathrm{p}_{1} \mathrm{~F}_{0}\left(\mathrm{p}_{0}, \mathrm{r}, \mathrm{q}_{2}, \mathrm{q}_{2}^{\prime}\right), \quad \mathrm{H}_{0}^{(1)}=\mathrm{p}_{1} \mathrm{~F}_{0}\left(\mathrm{p}_{0}, \mathrm{r}, \mathrm{q}_{2}, \mathrm{q}_{2}^{\prime}\right), \\
& \mathrm{H}_{0}^{(2)}=\mathrm{p}_{0} \mathrm{H}_{1}^{(2)}+\mathrm{p}_{1}^{2} \mathrm{~F}_{1}\left(\mathrm{p}_{0}, \mathrm{r}, \mathrm{q}_{2}, \mathrm{q}_{2}^{\prime}\right), \\
& \mathrm{H}_{1}^{(2)}=2 \mathrm{p}_{2} \mathrm{~F}_{1}\left(\mathrm{p}_{0}, \mathrm{r}, \mathrm{q}_{2}, \mathrm{q}_{2}^{\prime}\right)+\mathrm{p}_{1}^{2} \frac{\partial \mathrm{F}_{1}}{\partial \mathrm{p}_{0}}+\mathrm{p}_{1}\left(\frac{\mathrm{p}_{2}}{\mathrm{p}_{1}^{\prime}}-\frac{\mathrm{p}_{1}^{2} \mathrm{p}_{2}^{\prime}}{\mathrm{p}_{1}^{\prime 3}}\right) \frac{\partial \mathrm{F}_{1}}{\partial \mathrm{r}}+\mathrm{p}_{1} \mathrm{q}_{2} \frac{\partial \mathrm{F}_{1}}{\partial \mathrm{q}_{2}}+\frac{\mathrm{p}_{1}^{2} \mathrm{q}_{1}^{\prime}}{\mathrm{p}_{1}^{\prime}} \frac{\partial \mathrm{F}_{1}}{\partial \mathrm{q}_{2}^{\prime}},
\end{aligned}
$$

where $\mathrm{F}_{0}, \mathrm{~F}_{1}$ are arbitrary smooth functions of the variables $\mathrm{p}_{0}, \mathrm{r}=\mathrm{p}_{1} / \mathrm{p}_{1}^{\prime}, \mathrm{q}_{2}, \mathrm{q}_{2}^{\prime}$. Note that the corresponding biHamiltonian system reduces to a system of three equation for the two top order positions $\mathrm{q}_{2}, \mathrm{q}_{2}^{\prime}$, and the homogeneous momentum coordinate $r$; the remaining variables can be then determined by quadratures and solving linear systems. 
In general, as in the example, such biHamiltonian systems reduce to the integration of a $2 \mathrm{~m}-2$ dimensional Hamiltonian system for the coordinates $p_{1}^{i}, q_{n_{i}}^{i}, i=1, \ldots, m$, followed by a sequence of forced linear Hamiltonian systems. The final coordinate $q_{0}$ is determined by quadrature. Acutally, the initial Hamiltonian system can be reduced in order to $2 \mathrm{~m}-3$ since it only involves the homogeneous ratios of momenta $\mathrm{r}^{\mathrm{i}}=\mathrm{p}_{1}^{\mathrm{i}} / \mathrm{p}_{1}^{1}, \mathrm{i} \geq 2$, as can be seen from the formula for $\mu^{\mathrm{i}}$.

\section{The General Case.}

Theorem 15. Every generic non-degenerate, compatible Hamiltonian pair can be locally expressed as a Cartesian product of elementary (constant and non-constant eigenvalue) Hamiltonian pairs. Every associated biHamiltonian system decomposes into independent subsystems corresponding to each elementary subpair. Therefore the solution of the biHamiltonian system reduces to a collection of autonomous Hamiltonian systems whose dimensions are determined by the number of irreducible subpairs in each distinct elementary pair, along with a sequence of linear, non-autonomous Hamiltonian systems.

As demonstrated by Turiel, [14], [15], all these results have real counterparts, obtained by taking real and imaginary parts of the complex pairs corresponding to complex eigenvalues. In particular, as in [7], complex eigenvalue biHamiltonian systems are have necessarily analytic Hamiltonians. We note that Theorem 15 gives a refined version of the integrability results appearing in [4], [9]. Moreover, this result can be applied to give a more complete version of the result in [10], which demonstrates that the existence of enough biHamiltonian vector fields implies that the two Hamiltonian structures must be constant multiples of each other.

This completes our classification of compatible non-degenerate biHamiltonian systems. A significant open problem which has not been addressed is to extend these results to degenerate compatible Hamiltonian pairs.

\section{Hamiltonian Pairs in Four Dimensions.}

In 1946, Debever, [3], used Cartan's equivalence method, cf. [5], to classify pairs of symplectic two-forms on a four-dimensional complex manifold up to local (analytic) diffeomorphism. Debever does not impose any compatibility condition (which was not known at the time), but does impose an algebraic restriction, cf. (28) below; consequently the classification of Hamiltonian pairs in four dimensions is not complete. Nevertheless, 
Debever's result does produce new and interesting explicit examples of incompatible Hamiltonian pairs, and is worth reviewing in the context of Magri's Theorem.

Theorem 16. Let $\Omega_{1}, \Omega_{2}$ be analytic, symplectic two forms on a fourdimensional complex manifold $\mathrm{M}$ satisfying the algebraic condition

$$
\Omega_{1} \wedge \Omega_{2}=0 .
$$

Then there exist local coordinates $\left(\mathrm{p}_{1}, \mathrm{p}_{2}, \mathrm{q}_{1}, \mathrm{q}_{2}\right)$ such that

$$
\Omega_{1}=\mathrm{dp}_{1} \wedge \mathrm{dq}_{1}+\mathrm{dp}_{2} \wedge \mathrm{dq}_{2}
$$

and, up to constant multiple, $\Omega_{2}$ is equivalent to one of the three canonical forms

$$
\begin{aligned}
& \Omega_{2}^{(1)}=\mathrm{dp}_{1} \wedge \mathrm{dq}_{1}-\mathrm{dp}_{2} \wedge \mathrm{dq}_{2} \\
& \Omega_{2}^{(2)}=\mathrm{e}^{\mathrm{p}_{1}}\left\{\mathrm{dp}_{1} \wedge \mathrm{dq}_{1}-\mathrm{dp}_{2} \wedge \mathrm{dq}_{2}-\mathrm{p}_{2} \mathrm{dp}_{1} \wedge \mathrm{dq}_{2}\right\} \\
& \Omega_{2}^{(3)}=\mathrm{e}^{\mathrm{p}_{1}+\mathrm{p}_{2}}\left\{\mathrm{dp}_{1} \wedge \mathrm{dq}_{1}-\mathrm{dp}_{2} \wedge d \mathrm{~d}_{2}+\left(\mathrm{q}_{1}+\mathrm{q}_{2}\right) \mathrm{dp}_{1} \wedge \mathrm{dp}_{2}\right\}
\end{aligned}
$$

Moreover, the canonical symplectic two-form $\Omega_{1}$ is compatible with the symplectic twoform $\Omega_{2}^{(1)}$, but is not compatible with either $\Omega_{2}^{(2)}$ or $\Omega_{2}^{(3)}$.

Determining the general structure and integrability of biHamiltonian systems associated with these Hamiltonian pairs is not difficult. In the compatible case 1, the pair has two distinct constant eigenvalues, $\pm \lambda$. According to Theorem 15 , any biHamiltonian system for this pair decouples into a pair of autonomous planar Hamiltonian systems, and is thereby integrable, as can also be verified directly.

In case 2, the general biHamiltonian system has Hamiltonians of the general form

$$
\mathrm{H}_{1}=\mathrm{F}\left(\mathrm{p}_{2} \mathrm{e}^{\mathrm{p}_{1} / 2}, \mathrm{q}_{2}\right) \mathrm{e}^{-\mathrm{p}_{1} / 2}+\mathrm{g}\left(\mathrm{p}_{1}\right), \quad \mathrm{H}_{0}=-\mathrm{F}\left(\mathrm{p}_{2} \mathrm{e}^{\mathrm{p}_{1} / 2}, \mathrm{q}_{2}\right) \mathrm{e}^{\mathrm{p}_{1} / 2}+\hat{\mathrm{g}}\left(\mathrm{p}_{1}\right),
$$

where $\hat{\mathrm{g}}^{\prime}(\mathrm{s})=\mathrm{e}^{-\mathrm{s}} \mathrm{g}^{\prime}(\mathrm{s})$. The corresponding bi-Hamiltonian system is

$$
\begin{aligned}
& \frac{\mathrm{dp}_{1}}{\mathrm{dt}}=0, \quad \frac{\mathrm{dq}}{\mathrm{dt}}=\frac{1}{2} \mathrm{~F}\left(\mathrm{p}_{2} \mathrm{e}^{\mathrm{p}_{1} / 2}, \mathrm{q}_{2}\right) \mathrm{e}^{-\mathrm{p}_{1} / 2}-\frac{1}{2} \mathrm{p}_{2} \mathrm{~F}_{1}\left(\mathrm{p}_{2} \mathrm{e}^{\mathrm{p}_{1} / 2}, \mathrm{q}_{2}\right)+\mathrm{g}^{\prime}\left(\mathrm{p}_{1}\right), \\
& \frac{\mathrm{dp}_{2}}{\mathrm{dt}}=-\mathrm{F}_{2}\left(\mathrm{p}_{2} \mathrm{e}^{\mathrm{p}_{1} / 2}, \mathrm{q}_{2}\right) \mathrm{e}^{-\mathrm{p}_{1} / 2}, \quad \frac{\mathrm{dq}_{2}}{\mathrm{dt}}=\mathrm{F}_{1}\left(\mathrm{p}_{2} \mathrm{e}^{\mathrm{p}_{1} / 2}, \mathrm{q}_{2}\right)
\end{aligned}
$$


the subscripts on $\mathrm{F}$ indicating partial derivatives. Once the constant value of $\mathrm{p}_{1}$ is fixed, the integration of this system reduces to solving a single autonomous planar Hamiltonian system for $\mathrm{p}_{2}, \mathrm{q}_{2}$, with Hamiltonian function $\mathrm{f}\left(\mathrm{p}_{2} \mathrm{e}^{\mathrm{p}_{1} / 2}, \mathrm{q}_{2}\right) \mathrm{e}^{-\mathrm{p}_{1} / 2}$. The remaining function $\mathrm{q}_{1}$ can then be determined by a single quadrature.

In case 3 , the Hamiltonians have the general form

$$
\mathrm{H}_{0}=\mathrm{c}\left(\mathrm{q}_{1}-\mathrm{q}_{2}\right)+\mathfrak{\mathrm { f }}\left(\mathrm{p}_{1}, \mathrm{p}_{2}\right), \quad \mathrm{H}_{1}=\mathrm{c}\left(\mathrm{q}_{1}-\mathrm{q}_{2}\right)+\mathrm{f}\left(\mathrm{p}_{1}, \mathrm{p}_{2}\right)
$$

where $\mathrm{c}$ is a constant, and where

$$
\frac{\partial \widetilde{\mathrm{f}}}{\partial \mathrm{p}_{1}}=\mathrm{e}^{\mathrm{p}_{1}+\mathrm{p}_{2}} \frac{\partial \mathrm{f}}{\partial \mathrm{p}_{1}}, \quad \frac{\partial \widetilde{\mathrm{f}}}{\partial \mathrm{p}_{2}}=-\mathrm{e}^{\mathrm{p}_{1}+\mathrm{p}_{2}} \frac{\partial \mathrm{f}}{\partial \mathrm{p}_{2}}
$$

Note that the integrability condition for (33) implies that $f$ satisfies the partial differential equation $2 \mathrm{f}_{12}+\mathrm{f}_{1}+\mathrm{f}_{2}=0$. The associated bi-Hamiltonian system is

$$
\frac{\mathrm{dp}_{1}}{\mathrm{dt}}=-\mathrm{c}, \quad \frac{\mathrm{dp}_{2}}{\mathrm{dt}}=\mathrm{c}, \quad \frac{\mathrm{dq}}{\mathrm{dt}}=\frac{\partial \widetilde{\mathrm{f}}}{\partial \mathrm{p}_{1}}, \quad \frac{\mathrm{dq}}{\mathrm{dt}}=\frac{\partial \widetilde{\mathrm{f}}}{\partial \mathrm{p}_{2}},
$$

whose integration is trivial, reducing to just two quadratures.

Thus, by direct analysis, we are led to a strengthened version of Magri's Theorem for this particular case: Any four-dimensional biHamiltonian system satisfying the algebraic condition (28), compatible or not, is completely integrable. Indeed, the compatible biHamiltonian structure leads to systems which reduce to the two decoupled planar autonomous Hamiltonian systems, whereas for the incompatible pairs given by $\Omega_{2}^{(2)}$ and $\Omega_{2}^{(3)}$, the systems reduce to one planar Hamiltonian system and one quadrature, or just two quadratures, respectively. It would be extremely interesting to extend Debever's classification to all possible nondegenerate pairs of two forms on $\mathbb{C}^{4}$; this would go a long way to elucidating the precise role of the compatibility condition in the integrability of biHamiltonian systems ${ }^{\dagger}$. Indeed, based on the evidence so far, (including results on quasilinear hyperbolic systems, [12]) it would appear that incompatible biHamiltonian systems are, in a sense, even more integrable than compatible ones!

$\dagger$ This problem also has significant applications in I. Anderson's recent work on the Jesse Douglas inverse problem for second order systems, [1]. 
The proofs and further details on these results will appear in an expanded version to be published elsewhere. I would like to gratefully acknowledge the support of the Institute for Mathematics and its Applications (I.M.A.) during the time this work was completed. It is a pleasure to thank Darryl Holm, Niky Kamran, Yvette Kosmann-Schwarzbach, and Franco Magri, who all provided vital encouragement and alerted me to many of the important references in the subject. I would especially like to thank Francisco-Javier Turiel for sending me his classification results and alerting me to mistakes in an earlier version of this paper. 


\section{References}

[1] Anderson, I.M., personal communication.

[2] Arnol'd, V.I., Poisson structures on the plane and other powers of volume forms, J. Soviet Math. 47 (1989), 2509-2516.

[3] Debever, R., Quelques problèmes d'équivalence de formes différentielles alternées, Bull. Acad. Roy. de Belgique, Classe des Sciences 31 (1946), 262-277.

[4] De Fillipo, S., Vilasi, G., Marmo, G., Salerno, M., A new characterization of completely integrable systems, Il Nuovo Cim. 83B (1984), 97-112.

[5] Gardner, R.B., The Method of Equivalence and Its Applications, SIAM, Philadelphia, 1989.

[6] Gantmacher, F.R., The Theory of Matrices, vol. 2, Chelsea Publ. Co., New York, 1959.

[7] Jodeit, M. and Olver, P.J., On the equation grad f = M grad g, preprint, 1990.

[8] Magri, F., A simple model of the integrable Hamiltonian equation, J. Math. Phys. 19 (1978) 1156-1162.

[9] Magri, F., Alcune osservazioni sui sistemi hamiltoniani integrabili, preprint, "Solitons Study Group", I.C.T.P., Trieste, 1979.

[10] Marmo, G., Saletan, E.J., Schmid, R. and Simoni, A., Bi-Hamiltonian dynamical systems and the quadratic-Hamiltonian theorem, Il Nuovo Cim. $100 \mathrm{~B}$ (1987), 297-317.

[11] Olver, P.J., Applications of Lie Groups to Differential Equations, Graduate Texts in Mathematics, vol. 107, Springer-Verlag, New York, 1986.

[12] Olver, P.J. and Nutku, Y., Hamiltonian structures for systems of hyperbolic conservation laws, J. Math. Phys. 29 (1988), 1610-1619.

[13] Olver, P.J., A nonlinear series differential operator which commutes with any function, preprint, 1990.

[14] Turiel, F.-J., Classification locale d'un couple de formes symplectiques Poissoncompatibles, Comptes Rendus Acad. Sci. Paris 308 (1989), 575-578.

[15] Turiel, F.-J., Classification locale d'un couple de formes symplectiques compatibles, Séminaire de Géometrie et Topologie Différentiel de Montpellier, 1988-89, to appear. 
Li Ta-Tsien (Li Da-qian) and Zhao Yan-Chun, Global Existence of Classical Solutions to the Typical Free Boundary Problem for General Quasilinear Hyperbolic Systems and its Applications

Thierry Cazenave and Fred B. Weissler, The Structure of Solutions to the Pseudo-Conformally Invariant Nonlinear Schrödinger Equation

Marshall Slemrod and Athanasios E. Tzavaras, A Limiting Viscosity Approach for the Riemann Problem in Isentropic Gas Dynamics

Richard D. James and Scott J. Spector, The Formation of Filamentary Voids in Solids

P.J. Vassiliou, On the Geometry of Semi-Linear Hyperbolic Partial Differential Equations in the Plane Integrable by the Method of Darboux

Wenxiong Liu, A Parabolic System Arising In Film Development

Daniel B. Dix, Temporal Asymptotic Behavior of Solutions of the Benjamin-Ono-Burgers Equation

Michael Renardy and Yuriko Renardy, On the nature of boundary conditions for flows with moving free surfaces

Werner A. Stahel, Robust Statistics: From an Intellectual Game to a Consumer Product

Avner Friedman and Fernando Reitich, The Stefan Problem with Small Surface Tension

E.G. Kalnins and W. Miller, Jr., Separation of Variables Methods for Systems of Differential Equations in Mathematical Physics

Mitchell Luskin and George R. Sell, The Construction of Inertial Manifolds for Reaction-Diffusion Equations by Elliptic Regularization

Konstantin Mischaikow, Dynamic Phase Transitions: A Connection Matrix Approach

Philippe Le Floch and Li Tatsien, A Global Asymptotic Expansion for the Solution to the Generalized Riemann Problem

Matthew Witten, Ph.D., Computational Biology: An Overview

Matthew Witten, Ph.D., Peering Inside Living Systems: Physiology in a Supercomputer

Michael Renardy, An existence theorem for model equations resulting from kinetic theories of polymer solutions

589 Daniel D. Joseph and Luigi Preziosi, Reviews of Modern Physics: Addendum to the Paper "Heat Waves"

Luigi Preziosi, An Invariance Property for the Propagation of Heat and Shear Waves Time Effects

Prabir Daripa, On the Computation of the Beltrami Equation in the Complex Plane

Philippe Le Floch, Shock Waves for Nonlinear Hyperbolic Systems in Nonconservative Form

A.L. Gorin, D.B. Roe and A.G. Greenberg, On the Complexity of Pattern Recognition Algorithms On a Tree-Structured Parallel Computer

Mark J. Friedman and Eusebius J. Doedel, Numerical computation and continuation of invariant manifolds connecting fixed points

Scott J. Spector, Linear Deformations as Global Minimizers in Nonlinear Elasticity

Denis Serre, Richness and the classification of quasilinear hyperbolic systems

L. Preziosi and F. Rosso, On the stability of the shearing flow between pipes

Avner Friedman and Wenxiong Liu, A system of partial differential equations arising in electrophotography

600

Jonathan Bell, Avner Friedman, and Andrew A. Lacey, On solutions to a quasilinear diffusion problem from the study of soft tissue

601 David G. Schaeffer and Michael Shearer, Loss of hyperbolicity in yield vertex plasticity models under nonproportional loading

602 Herbert C. Kranzer and Barbara Lee Keyfitz, A strictly hyperbolic system of conservation laws admitting singular shocks

603 S. Laederich and M. Levi, Qualitative dynamics of planar chains

604

605

Milan Miklavčič, A sharp condition for existence of an inertial manifold

Charles Collins, David Kinderlehrer, and Mitchell Luskin, Numerical approximation of the solution of a variational problem with a double well potential

606 Todd Arbogast, Two-phase incompressible flow in a porous medium with various nonhomogeneous boundary conditions 
607 Peter Poláčik, Complicated dynamics in scalar semilinear parabolic equations in higher space dimension

608 Bei $\mathbf{H u}$, Diffusion of penetrant in a polymer: a free boundary problem

Mohamed Sami ElBialy, On the smoothness of the linearization of vector fields near resonant hyperbolic rest points

Max Jodeit, Jr. and Peter J. Olver, On the equation $\operatorname{grad} f=M \operatorname{grad} g$

611 Shui-Nee Chow, Kening Lu, and Yun-Qiu Shen, Normal form and linearization for quasiperiodic systems

Prabir Daripa, Theory of one dimensional adaptive grid generation

Michael C. Mackey and John G. Milton, Feedback, delays and the origin of blood cell dynamics

D.G. Aronson and S. Kamin, Disappearance of phase in the Stefan problem: one space dimension

Martin Krupa, Bifurcations of relative equilibria

616 D.D. Joseph, P. Singh, and K. Chen, Couette flows, rollers, emulsions, tall Taylor cells, phase separation and inversion, and a chaotic bubble in Taylor-Couette flow of two immiscible liquids

Artemio González-López, Niky Kamran, and Peter J. Olver, Lie algebras of differential operators in two complex variables

L.E. Fraenkel, On a linear, partly hyperbolic model of viscoelastic flow past a plate

Stephen Schecter and Michael Shearer, Undercompressive shocks for nonstrictly hyperbolic conservation laws

Xinfu Chen, Axially symmetric jets of compressible fluid

J. David Logan, Wave propagation in a qualitative model of combustion under equilibrium conditions

M.L. Zeeman, Hopf bifurcations in competitive three-dimensional Lotka-Volterra Systems

Allan P. Fordy, Isospectral flows: their Hamiltonian structures, Miura maps and master symmetries

Daniel D. Joseph, John Nelson, Michael Renardy, and Yuriko Renardy, Two-Dimensional cusped interfaces

Avner Friedman and Bei Hu, A free boundary problem arising in electrophotography

Hamid Bellout, Avner Friedman and Victor Isakov, Stability for an inverse problem in potential theory

Barbara Lee Keyfitz, Shocks near the sonic line: A comparison between steady and unsteady models for change of type

Barbara Lee Keyfitz and Gerald G. Warnecke, The existence of viscous profiles and admissibility for transonic shocks

P. Szmolyan, Transversal heteroclinic and homoclinic orbits in singular perturbation problems

Philip Boyland, Rotation sets and monotone periodic orbits for annulus homeomorphisms

Kenneth R. Meyer, Apollonius coordinates, the N-body problem and continuation of periodic solutions

Chjan C. Lim, On the Poincare-Whitney circuitspace and other properties of an incidence matrix for binary trees

K.L. Cooke and I. Györi, Numerical approximation of the solutions of delay differential equations on an infinite interval using piecewise constant arguments

Stanley Minkowitz and Matthew Witten, Periodicity in cell proliferation using an asynchronous cell population

M. Chipot and G. Dal Maso, Relaxed shape optimization: The case of nonnegative data for the Dirichlet problem

Jeffery M. Franke and Harlan W. Stech, Extensions of an algorithm for the analysis of nongeneric Hopf bifurcations, with applications to delay-difference equations

Xinfu Chen, Generation and propagation of the interface for reaction-diffusion equations

Philip Korman, Dynamics of the Lotka-Volterra systems with diffusion

Harlan W. Stech, Generic Hopf bifurcation in a class of integro-differential equations

Stephane Laederich, Periodic solutions of non linear differential difference equations

Peter J. Olver, Canonical Forms and Integrability of BiHamiltonian Systems

S.A. van Gils, M.P. Krupa and W.F. Langford, Hopf bifurcation with nonsemisimple 1:1 Resonance

R.D. James and D. Kinderlehrer, Frustration in ferromagnetic materials

Carlos Rocha, Properties of the attractor of a scalar parabolic P.D.E.

Debra Lewis, Lagrangian block diagonalization 